\title{
Sarcoma of Prostate of Myofibroblastic Origin
}

JCR Tarunkumar P Jain, Sachin A Bhujbal, Hemant R Pathak

Department of Urology, T.N.M.College and B.Y.L Nair Ch. Hospital, Mumbai,

Maharashtra, India.

\section{Abstract:}

Sarcoma of prostate is a rare tumor wherein entire literature is based on few case reports and isolated case series. We hereby report a case of prostatic sarcoma of myofibroblastic origin that had come to our hospital with review of literature.

Key words: Hematuria, Lower Urinary Tract Symptoms, Prostatic Neoplasms, Rhabdomyosarcoma, Sarcoma.

\section{Introduction}

Sarcoma of prostate is a rare tumor accounting to $0.1 \%-0.2 \%$ of prostatic malignancy. Majority of the case series/reports consists of rhabdomyosarcoma or leiomyosarcoma. Myofibroblastic origin of prostatic sarcoma is a rare entity. Generally myofibroblastic tumour is seen post inflammation, irradiation or instrumentation [1]. Inspite of multimodality treatment, prognosis of the patients is poor.

We are reporting this case of myofibroblastic sarcoma, its histopathology features, immunohistochemical findings, the radiological findings, the treatment he was subjected to and its outcome.

\section{Case Report}

57 years old male patient came to our institute with symptoms of acute urinary retention with history of lower urinary tract symptoms (LUTS) since 6 months. On examination his general condition was good and digital rectal examination revealed grade 2 prostate which was firm and had a smooth surface. Serum PSA was $3.74 \mathrm{ng} / \mathrm{mL}$. Ultrasonography revealed $90 \mathrm{cc}$ prostate with a large median lobe. Patient underwent TURP during which it was noted that the gland was excessively vascular. Patient was discharged on post-operative day 2 with successful catheter free trial.

However, patient came back with hematuria for which he had to be catheterized. The histopathology report showed fascicles of spindle cells with eosinophilic infiltrate and collagen production suggestive of prostatic sarcoma. Immunohistochemistry (IHC) was done which showed weak focal positivity for smooth muscle actin (SMA) and negative for $A E 1 / A E 3$, desmin, $\mathrm{H}$-caldesmon, CK-7 and CD-34, Mib-1 labeling index was 50\%.

Corresponding Author: Dr. Tarunkumar $P$ Jain

Email: tarunjain891986@gmail.com

Received: July 19, 2015 | Accepted: September 30, 2015 | Published Online: October 30, 2015

This is an Open Access article distributed under the terms of the Creative Commons Attribution License (creativecommons.org/licenses/by/3.0)

Conflict of interest: None declared | Source of funding: Nil | DOl: http://dx.doi.org/10.17659/01.2015.0119 
These findings pointed towards high grade sarcoma of myofibroblastic origin. Patient underwent whole body PET CT [Fig.1] which showed an irregular metabolically active mass in prostate invading base of bladder, another metabolically active solitary lesion was noted in middle lobe of right lung suggestive of metastasis [Fig.2].

Patient was planned for palliative chemotherapy with 3 cycles of ifosfamide and adriamycin. However after $1^{\text {st }}$ cycle patient developed febrile neutropenia and congestive cardiac failure due to which the decision to give no further chemotherapy was undertaken. After explaining the patient, he was subjected to definitive local radiotherapy to prostate at 60-65 Gy with weekly radiotherapy to lung lesion.

Patient improved symptomatically with completely settled hematuria. However, catheter free trial was not successful. Presently patient is 15 months into treatment and alive.

\section{Discussion}

Prostatic sarcoma is a rare malignant neoplasm that is believed to originate from specialized stromal prostatic cells. Gaudin et al. in 1998 was first to classify prostatic sarcoma into PSPUMP (prostatic stromal proliferation of unknown malignant potential) and PSS (prostatic stromal sarcoma) based on the degree of stromal cellularity and the presence of mitotic figures, necrosis, and stromal overgrowth [2]. He also suspected the progression of PSPUMP to PSS [2].

However, later on WHO in 2004 recognized stromal tumors as a different entity consisting of PSPUMP and PSS, separating it from mesenchymal tumors consisting of rhabdomyosarcomas, leiomyosarcomas etc. adding to the confusion. At present, there is still debate on its specific definition, pathology, and prognosis as many a time there are

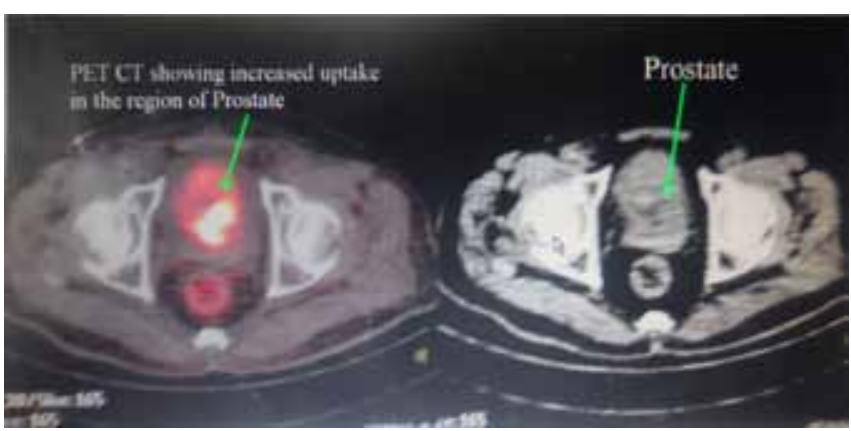

Fig.1: PET CT showing increased FDG uptake in prostatic area (arrow).

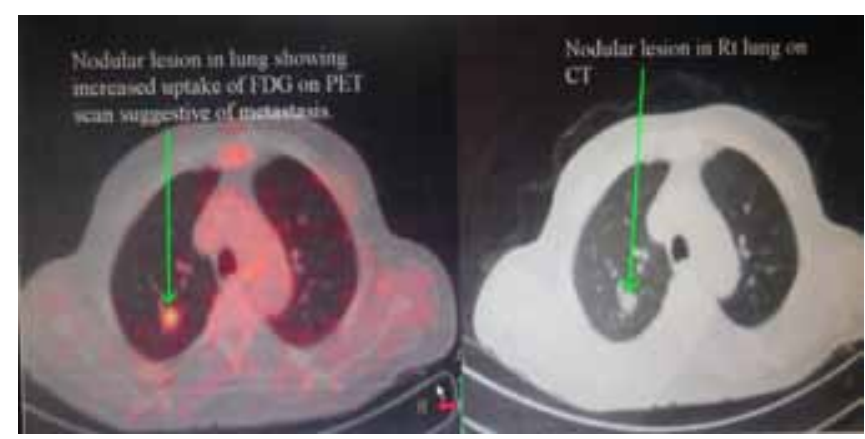

Fig.2: PET CT showing evidence of metastasis in lung with increased FDG uptake (arrow).

mixed features necessitating the need to clearly define these terminologies. Janet et al. classified prostate sarcoma into rhabdomyosarcoma (RMS) and non rhabdomyosarcoma (NRMS) for purpose of predicting prognosis [3]. Patients with RMS had a median survival of 142 months and NRMS with median survival of 24 months [3]. Hence, the need to identify the subtype.

$$
\text { Immunohistochemistry (IHC) plays a }
$$
very important role in classifying the tumor and identifying its origin. Stromal tumors (PSPUMP and PSS) are typically positive for CD34, vimentin and PR and negative for S-100 [2]. None of the cases classified as PSS during the analysis of a series of 22 cases by Gaudin et al. were positive for HHF35, SMA or desmin [2]. In a series of 23 cases of leiomyosarcoma, all cases were immunoreactive for 
vimentin, $63 \%$ were immunoreactive for actin, $20 \%$ were weakly reactive for desmin and S-100 protein was negative in all cases [4]. For myofibroblastic sarcoma, IHC lesions expressed anaplastic lymphoma kinase (ALK) in 57\%, AE $1 / 3$ in $73 \%$, CAM5.2 in $67 \%$, CK18 in $100 \%$, actin in $92 \%$, desmin in $79 \%$, calponin in $86 \%$, caldesmon in $57 \%$ and most lacked S100, CD34, CD1 17, CD21 and CD23 [1]. Based on their distinctive histological appearance and IHC profile, PSPUMP and PSS can be differentiated from other mesenchymal lesions of the prostate [2].

Treatment of prostatic sarcoma is extrapolated from the few case series/report and so is the efficacy of different approaches. Although it is universally agreed upon that early detection and surgical removal (radical prostatectomy or radical cystoprostatectomy) along with negative margin provides the best chance of cure $[5,7]$ but not only it is difficult to completely excise the tumor [4] but studies have shown multimodality treatment provides the best possible chance of long term recurrence free survival $[3,6]$. Chemotherapy for prostatic sarcoma is extrapolated from treatment protocols for soft tissue sarcoma elsewhere in the body. The same has been stated in the NCCN (National Comprehensive Cancer Network) guidelines version 1.2015. Majority of the regimens are based on combination of vincristine, adriamycin and cyclophosphamide/ ifosfamide.

Inspite of this the prognosis remains poor. The 5 year survival rate from various series ranges from $11.3 \%$ to $62.5 \%[5,6]$. Wang $X$ et al. found the survival for metastatic diseases was 1.5 years. Negative surgical margins and absence of metastatic disease at presentation were predictors of better survival [7]. Although RMS was associated with better prognosis as compared to NRMS [3], the same was not proven to be of statistical significance by Sexton et al [7]. Tumor size, grade and histological subtype of prostate sarcoma had no significant influence on survival [7]. Wang et al. in his studies proved that age $>50$ years, metastasis at presentation, and a lack of surgery with curative intent were independently predictive of an unfavorable outcome [5].

Local recurrences and distant metastasis are known to occur even after achieving negative margins. Patients have been reported to develop distant metastasis generally after 40 months of surgery most frequently to lungs and bones [4] again emphasizing the need for close monitoring and long term follow up [3].

\section{Conclusion}

Hence we would like to conclude by saying that there is still paucity of data on prostatic sarcoma, however with more and more cases being reported, in future we would be in a better position to optimize the treatment and predict the prognosis.

\section{References}

1. Montgomery EA, Shuster DD, Burkart AL, Esteban $J M$, Sgrignoli A, Elwood L, et al. Inflammatory myofibroblastic tumors of the urinary tract: a clinicopathologic study of 46 cases, including a malignant example inflammatory fibrosarcoma and a subset associated with high-grade urothelial carcinoma. Am J Surg Pathol. 2006;30(12):1502-1512.

2. Gaudin PB, Rosai J, Epstein JI. Sarcomas and related proliferative lesions of specialized prostatic stroma: a clinicopathologic study of 22 cases. Am J Surg Pathol. 1998;22(2):148-162.

3. Janet NL, May AW, Akins RS. Sarcoma of the prostate: a single institutional review. Am J Clin Oncol. 2009;32(1):27-29.

4. Cheville JC, Dundore PA, Nascimento AG, Meneses $M$, Kleer $E$, Farrow $G M$, et al. Leiomyosarcoma of the prostate. Report of 23 cases. Cancer. 1995;76(8):1422-1427. 
5. Wang X, Liu L, Tang H, Rao Z, Zhan W, Li X, et al. Twenty-five cases of adult prostate sarcoma treated at a high-volume institution from 1989 to 2009. Urology. 2013;82(1):160165.

6. Ball MW, Sundi D, Reese AC, Meyer CF, Terezakis $\mathrm{SA}$, Efron JE, et al. Multimodal Therapy in the
Treatment of Prostate Sarcoma: The Johns Hopkins Experience. Clin Genitourin Cancer. 2015;13(5):435-440.

7. Sexton WJ, Lance RE, Reyes AO, Pisters PW, Tu SM, Pisters LL. Adult prostate sarcoma: the $M$. D. Anderson Cancer Center Experience. J Urol. $2001 ; 166(2): 521-525$. 